\title{
EEG functional network properties related to visually induced unrecognized spatial disorientation
}

\author{
Yan $\mathrm{Li}^{\mathrm{a}}$, Yuanyuan Chen ${ }^{\mathrm{a}}$, Xiaoning Lv ${ }^{\mathrm{b}}$, Yang Yang ${ }^{\mathrm{a}}$, Jing Li ${ }^{\mathrm{b}}$, Chenru Hao ${ }^{\mathrm{a}}$, Minggao Li ${ }^{\mathrm{b}, *}$ \\ and Fang $\mathrm{Pu}^{\mathrm{a}, \mathrm{c}, *}$ \\ ${ }^{a}$ International Joint Research Center of Aerospace Biotechnology and Medical Engineering of \\ Ministry of Science and Technology of China, School of Biological Science and Medical Engineering, \\ Beihang University, No. 37 Xueyuan Road, Haidian District, Beijing 100191, P.R. China \\ ${ }^{b}$ Aviation and Nautical Medical Center, Navy General Hospital, No. 6 Fucheng Road, Haidian \\ District, Beijing 100048, P.R. China \\ ${ }^{c}$ State Key Laboratory of Virtual Reality Technology and Systems, Beihang University, No. 37 \\ Xueyuan Road, Haidian District, Beijing 100191, P.R. China
}

\begin{abstract}
Unrecognized spatial disorientation (SD) which is intimately linked with brain cognitive function is always a fatal issue for the safety of pilots. To explore its effects on human brain cognitive functions, electroencephalography (EEG) functional network analysis methods were adopted to examine topological changes in the connection of cognitive regions when experiencing unrecognized SD. Twelve male pilots participated in the study. They were subjected to a SD scene, namely visual rotation, which evoked unrecognized SD. For the main EEG frequency intervals, the phase lag index (PLI) and normalized mutual information (NMI) were calculated to quantify the EEG data. Then weighted connectivity networks were constructed and their properties were characterized in terms of an average clustering coefficient and global efficiency. A T-test was performed to compare PLI, NMI and network measures under unrecognized SD and non-SD conditions. It indicated a weak functional connectivity level in the theta band under unrecognized SD based on the significant decrease of mean values of PLI and NMI ( $\mathrm{p}<0.05)$. Meanwhile, both the average clustering coefficient and global efficiency in the theta band reduced under the unrecognized SD condition. The decrease of the average clustering coefficient and global efficiency demonstrates a lack of small-world characteristics and a decline in processing efficiency of brain cognitive regions. All the experimental results show that unrecognized SD may have a negative effect on brain functional networks in the theta band.
\end{abstract}

Keywords: Unrecognized spatial disorientation, cognitive function, EEG, network

\footnotetext{
${ }^{*}$ Address for correspondence: Minggao Li, Aviation and Nautical Medical Center, Navy General Hospital, No. 6 Fucheng Road, Haidian District, Beijing 100048, P.R. China. Tel.: +86-10-66958161; Fax: +86-10-66958161; E-mail: liminggao6899@163.com.

Fang Pu, International Joint Research Center of Aerospace Biotechnology and Medical Engineering of Ministry of Science and Technology of China, School of Biological Science and Medical Engineering, Beihang University, No. 37 Xueyuan Road, Haidian District, Beijing 100191, P.R. China. Tel.: +86-10-82338752; Fax: +86-10-82338752; E-mail: pufangbme@buaa.edu.cn.
} 


\section{Introduction}

With the development of aviation and space technology, astronauts and pilots are being sent into space on board various aircrafts and planes. Meanwhile, a series of problems is emerging, and spatial disorientation (SD) is one of these problems that have to be studied. During flight, three sensory systems named visual, vestibular and proprioceptive system often send conflicting information to the brain, and then three types of SD may happen, namely recognized SD, unrecognized SD and incapacitating SD [1]. SD often causes flying accidents, and statistics show that from $5 \%$ to $10 \%$ of all aviation accidents can be attributed to SD, while $90 \%$ of these accidents are fatal [2]. One thing to note is that accidents caused by unrecognized SD account for $80 \%$ of the total caused by SD [3].

The brain is considered to be a complex system, comprising spatially interconnected areas [4]. Similar with the connectivity of complex network, the structural and functional brain network possesses the small-world properties found by Watts and Strogatz [5]. It has been suggested that small-world properties support rapid adaptive reconfiguration of functional connectivity in response to varying cognitive demands [6] when listening to music [4] and learning [7]. Also many researches indicate that network measures show a great change in neurological diseases like Alzheimer's [8] and schizophrenia [9]. These findings indicate that the brain functional network has a dynamic feature according to different circumstances.

Research about SD on the ground concentrates on simulating various illusions by giving visual and vestibular stimuli. Visually evoked SD is easier to achieve on the ground [2, 10], the most well-understood type. One classic approach to produce this illusion is the rotation of one starry image applied by Tokumaru, et al. [10] in 1999. Eriksson, et al. [11] adopted visual flow (black background, white squares extend to the center). By applying EEG topographic map analyses method, Tokumaru, et al. [10] found a decreased potential in alpha band. Research of Stephens, et al. [12] showed that in black hole illusion, beta band waveform changed significantly and in Coriolis illusion, there was a significant change in theta band. Byung, et al. [13] explored the relationship between theta band and motion sickness and considered that theta band was an effective physiological parameter for motion sickness induced by visual stimuli. These researches are all focused on recognized SD. However, neither previous studies did make a distinction of recognized SD and unrecognized SD nor network analysis method has drawn enough attention in previous studies, thus this study applies EEG data in cognitive regions to construct functional network and intends to explore the effects of unrecognized SD on functional networks.

\section{Methods}

\subsection{Participants}

Twelve healthy pilots (mean age $26 \pm 1.65$ (SD) yrs) from one troop participated in the study. All the participants were right-handed, with normal vision and with no history of neurological or psychiatric disorders. The pilots were required to get enough sleep and not take any central nervous system drugs for three days before the test. For the posture balance test, the pilots were asked to keep their balance for 30 s with no obvious body sway.

\subsection{Apparatus and visual stimuli}

Apparatus in this experiment consist of a Neuroscan SynAmps2 (SynAmps2 ${ }^{\text {TM }}$ Model 8050 EEG amplifier and data acquisition system) with 64 electrodes, a plantar pressure force platform (Bertec 


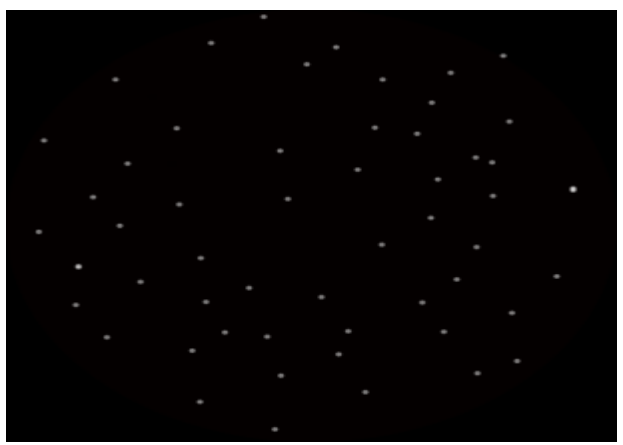

Fig. 1. Visual stimuli: visual rotation scene.

FP4060-08, Bertec Corp, Columbus, OH, USA), a projector (EPSON) and projection screen. In this experiment, the projection screen was placed $1 \mathrm{~m}$ in front of the force platform, and the pilots stood on the center of the platform with feet in a parallel position. The display on the projection screen subtended a visual angle of $78.2^{\circ} \times 62.8^{\circ}$.The refresh rate was $60 \mathrm{~Hz}$, and the resolution was $1024 \times$ 768 pixels. In addition, the experimental room was dark.

Visual stimuli consisted of one scene, namely visual rotation. As shown in Figure 1, the rotation scene was a rotated picture including 50 randomly distributed white circular dots at $60 \%$ rotation speed. Two directions of rotation were displayed (clockwise and anticlockwise).

According to the visual stimuli, two experiment sessions were designed (clockwise rotation and anticlockwise rotation).In each session, every pilot underwent 6 trials (3 static control, 3 experimental tests, the corresponding static image was used as static control conditions), viewing the stimuli for $30 \mathrm{~s}$ during each trial and was asked to report their subjective judgments about the emergence of SD, which was expressed by the feeling of self-rotation.

\subsection{Data acquisition and preprocessing}

When the subjects stood on the force platform, the Center of Pressure (COPx and COPy) were recorded with a $100 \mathrm{~Hz}$ low pass filter, at a $1000 \mathrm{~Hz}$ sampling frequency. Then the data were filtered by a $10 \mathrm{~Hz}$ Butterworth low pass filter.

According to previous EEG studies of cognition, we chose electrodes of functional regions related to cognition [14-16] to record the EEG, including FPz, Fz, F3, F4, F7, F8, Cz, C3, C4, T7, T8, Pz, P3, P4, P7, P8, POz, Oz, O1, O2. In addition, horizontal and vertical electrooculograms (HEOG, VEOG) were recorded from two bipolar electrodes placed slightly lateral to the outer canthus of each eye and above and below the left eye. All the channels were re-referenced to two mastoid channels, namely M1 and M2. Impedance values of all electrodes were kept below $10 \mathrm{~K} \Omega$. Then EEG was continuously recorded within a bandwidth of $0.05 \sim 100 \mathrm{~Hz}$, at a $500 \mathrm{~Hz}$ sampling rate. Ocular artifacts were removed by means of a regression approach [17] implemented in Scan 4.5, and the signal of each channel was decomposed into delta $(0-3.5 \mathrm{~Hz})$, theta $(4-7 \mathrm{~Hz})$, alpha $(8-13 \mathrm{~Hz})$, beta $(14-30 \mathrm{~Hz})$ and gamma $(31-45 \mathrm{~Hz})$ bands.

\subsection{Unrecognized $S D$}

As we know, spatial orientation needs the integration of information of visual, vestibular and proprioceptive system and $80 \%$ of the information comes from visual system [1]. When visual 
information conflicts with vestibular and proprioceptive information, an erroneous sense of position and motion (SD) may appear. As viewing a moving visual field, information based on G-force (vestibular and proprioception) would be suppressed relative to visual information, then the erroneous perception of position induces posture sway [18], so this performance is in consistency with spatial disorientation and can act as a typical feature of SD. Posture sway can be represented by the offset of COP, like the study of Ehrenfried, et al. [19], it indicated a significant increase of COP sway path length during the exposure to visual motion and Thurrel, et al. [20] also found that subjects experiencing visually evoked SD had a large offset of COP. Accordingly, in this study, root mean square error (RMSE) was calculated to measure the offset of COP, which indicated the arising of SD. For the visual stimuli, RMSE of COP in the control trials was computed to get the $95 \%$ confidence interval. Then, RMSE of COP in experimental trials were compared with the upper limit of the confidence interval, and when the RMSE data fell outside the upper limit, it indicated the emergence of SD. Unrecognized SD denotes that the pilots are unaware that they are disoriented, with the subjects' subjective judgments and objective offset of COP, for this research, unrecognized SD could be sifted out when subjective judgment expressed non-SD, but a large offset of COP appeared during the trial. The determining method was shown in Table 1.

\subsection{Phase lag index}

The phase lag index (PLI) was chosen to quantify synchronization between any pairwise combination of the 20 EEG channels, because it is much less affected by the influence of common resources and active reference electrodes [21]. Both the instantaneous amplitude $A(t)$ and the instantaneous phase can be computed by Eq. (1) $(H[x(t)]$ is the Hilbert transformation of signal $x(t))$ :

$$
A(t)=\sqrt{[x(t)]^{2}+[H[x(t)]]^{2}} \text { and } \varphi(t)=\arctan \frac{H[x(t)]}{x(t)}
$$

According to the phase of two EEG signals $x_{i}(t)$ and $x_{j}(t)$, the skewing can be obtained as

$$
\varphi_{i j}(t)=\varphi_{i}(t)-\varphi_{j}(t)
$$

Table 1

Determining methods of unrecognized SD

\begin{tabular}{llll}
\hline Number & Subjective judgment & RMSE significant difference & Result \\
\hline 1 & Y & Y & Recognized SD \\
2 & Y & N & Recognized SD \\
3 & N & Y & Unrecognized SD \\
4 & N & N & Non-SD \\
\hline
\end{tabular}

Note: Subjective judgment: Y represents experiencing SD; N represents experiencing non-SD. RMSE significant difference: $Y$ represents the RMSE of COP falls outside the upper limit, which indicates the happening of SD; N represents the RMSE data falls inside the upper limit, which indicates non-SD.

From the phase difference, the PLI is defined as an asymmetry measure for the phase difference 
distribution obtained from a time series of phase differences, $\mathrm{k}=1 \ldots \mathrm{N}$ :

$$
P L I_{i j}=\left|\left\langle\operatorname{sign}\left(\varphi_{i j}\left(t_{k}\right)\right)\right\rangle\right|
$$

$\mathrm{N}$ denotes the number of sampling points. The value of $P L I_{i j}$ is restricted between 0 and 1 . A $P L I_{i j}$ of 0 indicates no connection between two nodes. A $P L I_{i j}$ of 1 indicates strong connectivity between any two nodes.

\subsection{Normalized mutual information}

Mutual information (MI) can show the interaction function of different brain regions from the perspective of information transmission, as it is an entropy based on information theory. The normalized mutual information (NMI) value was limited to $0-1$ based on the transformation of MI. The NMI can reflect the integrated information of amplitude and phase. For two Random Variables $X$ $\left\{\mathrm{x}_{1}, \mathrm{x}_{2}, \mathrm{x}_{3}, \ldots \mathrm{x}_{\mathrm{N}}\right\}$ and $Y\left\{\mathrm{y}_{1}, \mathrm{y}_{2}, \mathrm{y}_{3}, \ldots \mathrm{y}_{\mathrm{N}}\right\}$, the united entropy is defined as:

$$
E(X, Y)=\sum_{i=1}^{N} \sum_{j=1}^{N} p_{i j} \log _{2} p_{i j}
$$

$p_{i j}$ denotes the joint probability of $\mathrm{x}_{\mathrm{i}}$ and $\mathrm{x}_{\mathrm{j}}$.

Then the mutual information of $X$ and $Y$ is described as:

$$
M I(X, Y)=E(X)+E(Y)-E(X, Y)
$$

For two EEG signals $x_{i}(t)$ and $x_{j}(t)$, the NMI can be calculated as:

$$
N M I\left(x_{i}(t), x_{j}(t)\right)=\frac{2 M I\left(x_{i}(t), x_{j}(t)\right)}{E\left(x_{i}(t)\right)+E\left(x_{j}(t)\right)}
$$

The value of $N M I$ is restricted between 0 and 1 . A $N M I$ of zero indicates no interaction between two channels during information transfer. A NMI of 1 indicates strong interaction between the channels.

\subsection{Brain network construction and analysis}

A complex brain network consists of a series of vertices that are linked by edges, the edges denote the associations between vertices. In this study, vertices refer to the available brain regions corresponding to 20 EEG channels, and edges refer to the value matrices of PLI and NMI for all pairs of EEG electrodes. That is, $20 * 20$ correlation matrices were constructed, the value of each matrix element denoted the edge weight between two vertices, which were represented by the row and column number of elements.

The PLI and NMI values were averaged over all pair-wise combinations of EEG electrodes to 

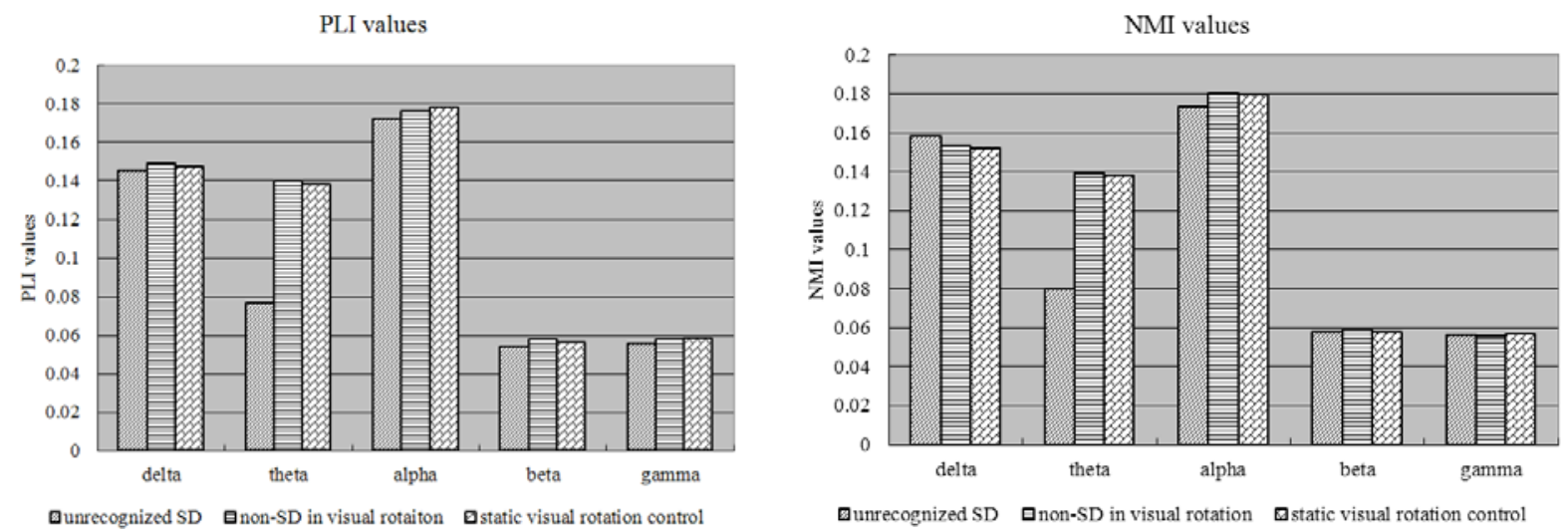

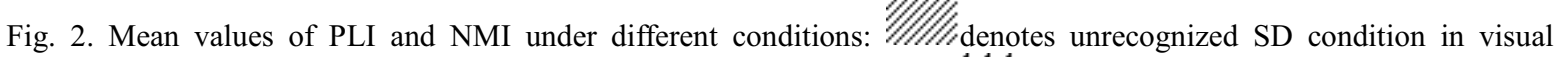
rotation scene, $\overline{\bar{\equiv}}$ denotes non-SD condition in visual rotation scene, $\not / 2 /$ denotes non-SD in static visual rotation scene.

demonstrate the mean levels of phase synchronization and information transmission efficiency. Then the clustering coefficient $\mathrm{C}^{\mathrm{w}}$ and the global efficiency $\mathrm{E}^{\mathrm{w}}$ were utilized to quantify the topological properties of weighted EEG functional networks based on PLI and NMI. The calculation of $\mathrm{C}^{\mathrm{w}}$ and $\mathrm{E}^{\mathrm{w}}$ can be referred to [4]. Then, the T-test $(\mathrm{p}=0.05)$ was adopted to compare the mean PLI and NMI values and the network measures $\mathrm{C}^{\mathrm{w}}$ and $\mathrm{E}^{\mathrm{w}}$ to disclose some network features in unrecognized SD.

\section{Results}

In visual rotation experiment group, of all 72 experiment trials, SD, including recognized SD and unrecognized SD accounted for 23 trials and among them, there were 16 unrecognized SD trials and the rotation direction does not make any significant difference.

Statistical analysis of mean PLI and mean NMI under unrecognized SD condition and non-SD condition, and the static condition (the trials served as baseline to calculate RMSE of COP) in five different frequency bands were performed using the T-test. As shown in Figure 2, data in non-SD related to two types of conditions (visual rotation and static visual rotation) showed no significant difference and the synchronization levels and mutual information levels in the theta band were significantly lower while experiencing unrecognized SD by viewing rotation scene.

Tables 2 and 3 show the changes of clustering coefficient and global efficiency calculated according to PLI and NMI. It showed no difference between non-SD in visual rotation and static visual rotation conditions. Both $\mathrm{C}^{\mathrm{w}}$ and $\mathrm{E}^{\mathrm{w}}$ had statistically significant differences in the theta frequency band. The T-test indicated that $\mathrm{C}^{\mathrm{w}}$ and $\mathrm{E}^{\mathrm{w}}$ were lower in unrecognized $\mathrm{SD}$ evoked by the rotation scene than in non-SD in visual rotation and static visual rotation control trials in the theta band.

\section{Discussion}

In this study, a network analysis approach was used to investigate changes in the topological properties of unrecognized SD. Using network analysis, the results revealed a series of decreases in 
Table 2

Mean values of clustering coefficient $\mathrm{C}^{\mathrm{w}}$ under different conditions

\begin{tabular}{llll}
\hline & Unrecognized SD & Non-SD in visual rotation & Static visual rotation \\
\hline $\mathrm{C}^{\mathrm{w}}(\mathrm{PLI})$ & Mean $\pm \mathrm{SD}$ & Mean $\pm \mathrm{SD}$ & Mean $\pm \mathrm{SD}$ \\
delta & $0.1267 \pm 0.0139$ & $0.1354 \pm 0.0071$ & $0.1274 \pm 0.0291$ \\
theta & $0.0776 \pm 0.0213 \boldsymbol{\nabla}^{\mathbf{1}} \boldsymbol{\nabla}^{\mathbf{2}}$ & $0.1223 \pm 0.0132$ & $0.1210 \pm 0.0138$ \\
alpha & $0.1493 \pm 0.0197$ & $0.1508 \pm 0.0335$ & $0.1527 \pm 0.0276$ \\
beta & $0.0478 \pm 0.0037$ & $0.0453 \pm 0.0033$ & $0.0489 \pm 0.0032$ \\
gamma & $0.0397 \pm 0.0055$ & $0.0416 \pm 0.0036$ & $0.0420 \pm 0.0064$ \\
$\mathrm{C}^{\mathrm{w}}(\mathrm{NMI})$ & Mean $\pm \mathrm{SD}$ & Mean $\pm \mathrm{SD}$ & Mean $\pm \mathrm{SD}$ \\
delta & $0.1363 \pm 0.0139$ & $0.1332 \pm 0.0071$ & $0.1326 \pm 0.0191$ \\
theta & $0.0795 \pm 0.0076 \boldsymbol{\nabla}^{\mathbf{1}} \mathbf{\nabla}^{\mathbf{2}}$ & $0.1342 \pm 0.0168$ & $0.1393 \pm 0.0164$ \\
alpha & $0.1685 \pm 0.0247$ & $0.1706 \pm 0.0152$ & $0.1692 \pm 0.0376$ \\
beta & $0.0539 \pm 0.0037$ & $0.0581 \pm 0.0038$ & $0.0537 \pm 0.0075$ \\
gamma & $0.0523 \pm 0.0055$ & $0.0548 \pm 0.0036$ & $0.0590 \pm 0.0034$ \\
\hline
\end{tabular}

Note: Mean values of $\mathrm{C}^{\mathrm{w}}$ calculated by PLI and NMI value in five frequency bands. $\nabla^{1}$ means statistically significant difference in unrecognized SD condition compared with non-SD in visual rotation condition; $\boldsymbol{\nabla}^{2}$ means statistically significant difference in unrecognized SD condition compared with static visual control.

Table 3

Mean values of global efficiency $\mathrm{E}^{\mathrm{w}}$ under different conditions

\begin{tabular}{llll}
\hline & Unrecognized SD & Non-SD in Visual rotation & Static visual rotation \\
\hline $\mathrm{E}^{\mathrm{w}}(\mathrm{PLI})$ & Mean $\pm \mathrm{SD}$ & Mean $\pm \mathrm{SD}$ & Mean $\pm \mathrm{SD}$ \\
delta & $0.1524 \pm 0.0157$ & $0.1527 \pm 0.0096$ & $0.1590 \pm 0.0118$ \\
theta & $0.1085 \pm 0.0201 \boldsymbol{\nabla}^{\mathbf{1}} \boldsymbol{\nabla}^{\mathbf{2}}$ & $0.1501 \pm 0.0174$ & $0.1503 \pm 0.0135$ \\
alpha & $0.1853 \pm 0.0272$ & $0.1935 \pm 0.0572$ & $0.1919 \pm 0.0387$ \\
beta & $0.0725 \pm 0.0087$ & $0.0783 \pm 0.0046$ & $0.0779 \pm 0.0053$ \\
gamma & $0.0641 \pm 0.0104$ & $0.0701 \pm 0.0040$ & $0.0631 \pm 0.0073$ \\
$\mathrm{E}^{\mathrm{w}}(\mathrm{NMI})$ & Mean $\pm \mathrm{SD}$ & Mean $\pm \mathrm{SD}$ & Mean $\pm \mathrm{SD}$ \\
delta & $0.1633 \pm 0.0131$ & $0.1647 \pm 0.0357$ & $0.1676 \pm 0.0318$ \\
theta & $0.1091 \pm 0.0517 \boldsymbol{\nabla}^{\mathbf{1}} \boldsymbol{\nabla}^{\mathbf{2}}$ & $0.1548 \pm 0.0423$ & $0.1537 \pm 0.0150$ \\
alpha & $0.1907 \pm 0.0236$ & $0.2011 \pm 0.0329$ & $0.1996 \pm 0.0380$ \\
beta & $0.0798 \pm 0.0036$ & $0.0834 \pm 0.0035$ & $0.0821 \pm 0.0064$ \\
gamma & $0.0800 \pm 0.0015$ & $0.0818 \pm 0.0044$ & $0.0804 \pm 0.0023$ \\
\hline
\end{tabular}

Note: Mean values of $\mathrm{E}^{\mathrm{w}}$ calculated by PLI and NMI value in five frequency bands. $\boldsymbol{\nabla}^{1}$ means statistically significant difference in unrecognized SD condition compared with non-SD in visual rotation condition; $\boldsymbol{\nabla}^{2}$ means statistically significant difference in unrecognized $\mathrm{SD}$ condition compared with static visual rotation control

EEG functional network properties related to unrecognized SD. First, the decrease of mean values of PLI and NMI in the unrecognized SD condition indicated a weaker functional connectivity level in the theta band. EEG alpha and theta waves are thought to reflect a person's cognitive and memory ability [22], and as visually evoked SD can provoke motion sickness, most studies found that theta band was an effective parameter to measure visually induced motion sickness, such as that Byung, et al. [13] found that there was the highest correlation between parameter of theta and subjective report of visual SD and motion sickness, similarly, in the study of Park, et al. [23], theta band was considered an effective physiological parameter for evaluating visually induced motion sickness and they also found different subject groups (sick and nonstick of motion sickness) were statistically different with respect 
to theta band at frontal lobe and parietal lobe; based on this, we guessed that unrecognized SD may have an adverse effect on pilots' cognitive function. In previous studies about cognition, Koenig, et al. [24] and Lee, et al. [25] found that patients with Alzheimer's disease had a weaker functional connectivity level in the beta and gamma bands. Subsequently, J.Q. Zhao, et al. [26] conducted an EEG functional network study of elderly people with mild cognitive impairment and found a decrease of connectivity in the theta band. All of these studies were focused on cognitive disorders that showed, on the one hand, that there may have some similar brain network performance characteristics between unrecognized SD and mild cognitive impairment, but on the other hand, after all, unrecognized SD is not a cognitive disorder with pathological EEG waveform and is just a temporary orientation impairment. Thus more scrutinization is needed before any conclusion could be drawn.

Next, a brain functional network was constructed and some network topological parameters like $\mathrm{C}^{\mathrm{w}}$ and $\mathrm{E}^{\mathrm{w}}$ were calculated to disclose some characteristics like small-world configurations. The study of $\mathrm{J}$. $\mathrm{Wu}$, et al. [4] revealed that human's brain functional network connectivity and small-world properties dynamically changed depending on the environment. The study by Ahmadlou, et al. [27] found that in the working memory task, there was a significant decrease of brain network complexity expressed by a lower clustering coefficient and global efficiency in patients with mild cognitive impairment in the theta and alpha bands. A larger global clustering coefficient indicates more specific function complied by different brain regions and a larger global efficiency demonstrates more efficient transmission of information among different functional regions. In this study, subjects under the non-SD condition had a greater clustering coefficient and global efficiency relative to the unrecognized SD condition. This may demonstrate that, compared with the non-SD condition, subjects in the unrecognized SD condition showed a lack of small-world characteristics. Like the research of Byung, et al. [13], as exposed to visual motion, when motion sickness appeared, subjects may become displeased and upset, in such conditions, the brain may have weaker ability to process and integrate information. We guessed that under displeasing conditions induced by viewing visual stimuli, pilots' brain functional networks had weaker connectivity, following this, the information processing and transfer capabilities of the pilots' brains became lower, and this may affect the spatial orientation capability and thus threaten safety.

In this research, the incidence of SD (recognized SD and unrecognized SD) was $32 \%$ and the induction rate of unrecognized SD was only $22.2 \%$. The occurrence ratio of SD was a little low, and this might be brought out by the short duration of the trials. Accordingly, concerning the future studies, longer duration of each trial may be taken into account to get greater induction rate of SD and unrecognized SD and more precise results.

\section{Conclusion}

This study finds some differences of brain functional networks in unrecognized SD by calculating the values of PLI and NMI and network measures like $\mathrm{C}^{\mathrm{w}}$ and $\mathrm{E}^{\mathrm{w}}$. All the results indicated that unrecognized SD may have a negative effect on human brain functional networks represented by the weakening connection capability of pilots' brain networks in the theta band. Further studies are needed to reveal more features of pilots' brain functional networks in spatial disorientation.

\section{Acknowledgment}


The authors wish to thank all the subjects who participated in the research, and acknowledge the financial supports from the Key Scientific Research Projects on Military Logistics (Grant No. AHJ2011Z001), and the Innovation Foundation of BUAA for PhD Graduates

\section{References}

[1] D.G. Newman, An overview of spatial disorientation as a factor in aviation accidents and incidents, Australia: Australian Transport Safety Bureau, Aviation Research and Analysis Report, B2007/0063, 2007.

[2] M.J. Antunano, Spatial disorientation: visual illusions, Medical Facts for Pilots, Federal Aviation Administration, 2013.

[3] W. Bles, Spatial disorientation training-demonstration and avoidance, Netherlands: The Research and Technology Organisation of North Atlantic Treaty Organisation Technical Report, TR- HFM-118, 2008.

[4] J. Wu, J. Zhang, X. Dong, R. Li and C. Zhou, The effects of music on brain functional networks: A network analysis, Neuroscience 250 (2013), 49-59.

[5] D.J. Watts and S.H. Strogatz, Collective dynamics of 'small-world' networks, Nature 393 (1998), 440-445.

[6] D.S. Bassett, A. Meyer-Lindenberg, S. Achard, T. Duke and E. Bullmore, Adaptive reconfiguration of fractal small-world human brain functional networks, Proceedings of the National Academy of Sciences of the United States of America 103 (2006), 19518-19523.

[7] D.S. Bassett, N.F. Wymbs, M.A. Porter, P.J. Mucha, J.M. Carlson and S.T. Grafton, Dynamic reconfiguration of human brain networks during learning, Proceedings of the National Academy of Sciences of the United States of America 108 (2011), 7641-7646.

[8] C.J. Stam, W. De Haan, A. Daffertshofer, B.F. Jones, I. Manshanden, A.M. van Cappellen van Walsum, T. Montez, J.P.A. Verbunt, J.C. de Munck, B.W. van Dijk, H.W. Berendse and P. Schelten, Graph theoretical analysis of magnetoencephalographic functional connectivity in Alzheimer's disease, Brain 132 (2009), 213-224.

[9] S. Micheloyannis, E. Pachou and C. Stam, Small-world networks and disturbed functional connectivity in schizophrenia, Schizophrenia Research 87 (2006), 60-66.

[10] O. Tokumaru, K. Kaida and H. Ashida, EEG topographical analysis of spatial disorientation, Aviation, Space, and Environmental Medicine 70 (1999), 256-263.

[11] L. Eriksson, C. Von Hofsten, A. Tribukait, O. Eiken, P. Ersson and J. Hedstrom, Visual flow scene effects on the somatogravic illusion in non-pilots, Aviation, Space, and Environmental Medicine 79 (2008), 860-866.

[12] M.S. Stephens, Electroneurophysiologic diagnosis of aircraft pilot spatial disorientation, Ph.D. Dissertation, Wright State University, 2003.

[13] C.M. Byung, C.C. Soon, K.M. Yoon and S. Kazuyoshi, Psychophysiological evaluation of simulator sickness evoked by a graphic simulator, Applied Ergonomics 35 (2004), 549-556.

[14] V.D. Constantinescu, Revealing the distinction between perception and cognition through intra-individual variability of visual evoked responses, International Journal of Bio-Medical Computing 40 (1996), 169-178.

[15] N.A. Busch, J. Dubois and R. Van Rullen, The phase of ongoing EEG oscillations predicts visual perception, The Journal of Neuroscience 29 (2009), 7869-7876.

[16] T. Ergenoglu, T. Demiralp and Z. Bayraktaroglu, Alpha rhythm of the EEG modulates visual detection performance in humans, Cognitive Brain Research 20 (2004), 376-383.

[17] H.V. Semlisch, P. Anderer, P. Schuster and O. Presslich, A solution for reliable and valid reduction of ocular artifacts applied to the P300 ERP, Psychophysiology 23 (1986), 695-703.

[18] L. Eriksson and C. Von Hofsten, Effects of visual flow display of flight maneuvers on perceived spatial orientation, Human Factors and Ergonomics Society 47 (2005), 378-393.

[19] T. Ehrenfried, M. Guerraz, K.V. Thilo, L. Yardley and M.A. Gresty, Posture and mental task performance when viewing a moving visual field, Cognitive Brain Research 17 (2003), 140-153.

[20] A. Thurrell and A. Bronstein, Vection increases the magnitude and accuracy of visually evoked postural responses, Experimental Brain Research 147 (2002), 558-560.

[21] C.J. Stam, G. Nolte and A. Daffertshofer, Phase lag index: assessment of functional connectivity from multi-channel EEG and MEG with diminished bias from common sources, Human Brain Mapping 28 (2007), 1178-1193.

[22] W. Klimesch, EEG alpha and theta oscillations reflect cognitive and memory performance: A review and analysis, Brain Research Reviews 29 (1999), 169-195.

[23] J.R. Park, D.W. Lim, H.W. Lee, M.H. Choi and S.C. Chunq, Long term study of simulator sickness: differences in EEG response due to individual sensitivity, International Journal of Neuroscience 118 (2008), 857-865.

[24] T. Koenig, L. Prichep, T. Dierks, D. Hubl, L.O. Wahlund, E.R. John and V. Jelic, Decreased EEG synchronization in 
Alzheimer's disease and mild cognitive impairment, Neurobiology of Aging 26 (2005), 165-171.

[25] S.H. Lee, Y.M. Park, D.W. Kim and C.H. Im, Global synchronization index as a biological correlate of cognitive decline in Alzheimer's disease, Neuroscience Research 66 (2010), 333-339.

[26] J.Q. Zhao, Y. Gao, L. Wei, J. Yang and Y.J. Li, The abnormality of brain functional connection in theta rhythm of patients with cognitive disorders, Acta Biophysica Sinica 30 (2014), 369-379.

[27] M. Ahmadlou, A. Adeli, R. Bajo and H. Adeli, Complexity of functional connectivity networks in mild cognitive impairment subjects during a working memory task, Clinical Neurophysiology 125 (2014), 694-702. 